Bio - grafía. Escritos sobre la Biología y su Enseñanza. ISSN 2027-1034

Edición Extraordinaria. p.p. $771-779$

Memorias del IX Encuentro Nacional de Experiencias en Enseñanza de la Biología y la Educación Ambiental. IV Congreso Nacional de Investigación en Enseñanza de la Biología.

\title{
CONCEPCIONES DEL PROFESORADO DE BIOLOGÍA SOBRE EVOLUCIÓN BIOLÓGICA DESDE EL PERFIL CONCEPTUAL.
}

\author{
Germán Alberto Chaves Mejia ${ }^{1}$
}

\section{RESUMEN}

El presente escrito muestra los resultados parciales de la investigación doctoral "Concepciones y prácticas del profesorado de biología sobre evolución biológica desde el perfil conceptual" en el marco del Doctorado Interinstitucional en Educación (DIE). Este escrito en términos generales tiene como propósito generar nuevo conocimiento sobre las concepciones del profesorado en torno al concepto Evolución Biológica desde los presupuestos teórico-metodológicos del Perfil Conceptual planteados por Mortimer y El Hani (2014). Los aspectos metodológicos se enmarcan en el paradigma cualitativo y se desarrollaron en una muestra de docentes amplia, heterogénea y no probabilística que enseñan Biología en Colombia.

En este orden de ideas, se construyó un Perfil Conceptual estableciendo categorías de análisis sobre los compromisos ontológicos y epistemológicos del profesorado al enseñar EB, los cuales desde una adaptación a lo planteado por Sepúlveda (2009) pudo evidenciar cuatro zonas de perfil para comprender las concepciones del profesorado estudiado, las cuales en orden de complejidad ascendente son, ajuste providencial, funcionalismo intra-orgánico, perspectiva transformacional y perspectiva variacional

Los aportes de esta investigación pueden constituirse en una nueva fuente de nuevo y valioso conocimiento en Didáctica de las Ciencias Naturales debido a que no han sido muy exploradas las concepciones de los docentes sobre un concepto científico clave, central, interdisciplinar y estructurante en las ciencias escolares como lo es la Evolución Biológica y mucho menos desde una perspectiva didáctica reciente, innovadora y de gran poder explicativo como la de los Perfiles Conceptuales.

PALABRAS CLAVE: Perfil Conceptual, Evolución Biológica, Enseñanza de la Biología, Concepciones del profesorado.

\section{ABSTRACT:}

This paper presents the partial results of the doctoral research "Conceptions and Practices of Biology Teachers on Biological Evolution from the Conceptual Profile" in the framework of the Interinstitutional Doctorate in Education (DIE). This paper, in general terms, aims to

\footnotetext{
${ }^{1}$ Estudiante del Doctorado Interinstitucional en Educación (DIE). Sede Universidad Distrital Francisco José de Caldas. Docente de la Secretaría de Educación de Bogotá.
} 


\section{Bio - grafía. Escritos sobre la Biología y su Enseñanza. ISSN 2027-1034}

Edición Extraordinaria. p.p. 771 - 779

Memorias del IX Encuentro Nacional de Experiencias en Enseñanza de la Biología y la Educación Ambiental. IV Congreso Nacional de Investigación en Enseñanza de la Biología.

generate new knowledge about teachers' conceptions around the concept of Biological Evolution from the theoretical-methodological assumptions of the Conceptual Profile proposed by Mortimer and El Hani (2014). The methodological aspects are framed in the qualitative paradigm and were developed in a sample of teachers, heterogeneous and non-probabilistic who teach Biology in Colombia.

In this context, a Conceptual Profile was constructed, establishing categories of analysis on the ontological and epistemological commitments of the teaching staff when teaching Biological Evolution, which from an adaptation to Sepúlveda (2009) could show four areas of profile to understand the Conceptions of the studied faculty, which in order of ascending complexity are providential adjustment, intra-organic functionalism, transformational perspective and variational perspective

The contributions of this research may constitute a new source of new and valuable knowledge in Didactics of the Natural Sciences due to the fact that teachers' conceptions of a key, central, interdisciplinary and structuring scientific concept in the school sciences have not been widely explored as it is the Biological Evolution and much less from a recent didactic perspective, innovative and of great explanatory power like the one of the Conceptual Profiles.

KEYWORDS: Conceptual Profile, Biological Evolution, Teaching of Biology, Conceptions of teachers.

\section{INTRODUCCIÓN}

El estudio de las concepciones del profesorado en contextos de enseñanza reviste un particular interés para la Didáctica de las Ciencias Naturales (DCN) en razón de que según Jimenez y Diaz de Bustamante (2003), Ogborn (1996) y Osborne Erduran, Dagher, Pintó y Couso (2007) los estudiantes en gran medida comprenden el mundo natural como este les ha sido enseñado, lo cual se basa en las ideas que tienen los docentes y que usualmente dan cuenta de sus prácticas de aula.

Es así como las concepciones de los docentes al enseñar conceptos científicos como lo es la Evolución Biológica (EB) puede constituirse en un aspecto clave en la DCN en razón de que la comprensión de la teoría de la EB y por tanto los procesos de enseñanza de la misma son fundamentales en la formación de ciudadanos científicamente alfabetizados que puedan comprender, transformar y tomar posiciones críticas frente cuestiones de diversa índole desde una posición integradora e interdisciplinar como el uso responsable y adecuado de antibióticos, el origen de las enfermedades (medicina), el manejo de plagas (agricultura), el origen del hombre y su lugar en el mundo (filosofía), la preservación de la biodiversidad, entre muchas otras.

Por otra parte, la teoría y la metodología de los Perfiles Conceptuales (PC) plantean nuevas perspectivas para comprender los conceptos como también su origen. Es así como los conceptos son tratados como procesos dinámicos construidos socialmente que 


\section{Bio - grafía. Escritos sobre la Biología y su Enseñanza. ISSN 2027-1034}

Edición Extraordinaria. p.p. 771 - 779

Memorias del IX Encuentro Nacional de Experiencias en Enseñanza de la Biología y la Educación Ambiental. IV Congreso Nacional de Investigación en Enseñanza de la Biología.

tienen su génesis desde elementos histórico-epistemológicos que determina sus contextos de uso.

La teoría de los PC emerge en 1995 en el campo de la educación en ciencias, a partir de la publicación en la revista Science \& Education del artículo "¿Cambio conceptual o cambio de perfil conceptual?" de autoría de Eduardo Mortimer. Luego de éste, se han realizado investigaciones, principalmente en Brasil, en las que la estructura de conceptos científicos relacionados con la vida, la materia y la energía son estudiados desde la base teórico-metodológica de los PC.

Una de los presupuestos principales sobre la teoría de los PC se basa en el hecho de que las personas pueden exhibir diferentes maneras de ver y representar el mundo en diferentes contextos, las cuales se despliegan en un perfil que permite un acercamiento para comprender el mundo, los cuales se pueden indagar estableciendo entre otras cosas los compromisos ontológicos y epistemológicos que poseen las personas con respecto a un concepto. Es así como esta perspectiva se constituye en una alternativa a lo propuesto por la teoría de cambio conceptual propuesta por Posner, Strike, Hewson y Gerzog (1982) que se caracteriza por proponer que, para generar un aprendizaje del conocimiento científico, los estudiantes deben abandonar y superar sus ideas previas y conocimiento cotidiano del mundo.

A continuación de una manera sucinta se desarrollaran aspectos teóricos, epistemológicos y metodológicos sobre los PC.

Los conceptos en la teoría de los PC: Una aproximación comúnmente utilizada sobre qué son los conceptos es la que los refiere como modelos mentales de aprendizaje de un objeto o evento. Desde esta perspectiva, los conceptos son internos, estables y pertenecientes al individuo.

Otra perspectiva sobre la naturaleza de los conceptos, la cual es adoptada por la teoría de los PC, propone, que los conceptos existen únicamente como parte de un lenguaje natural o de un sistema estructurado de conocimientos (como por ejemplo las ciencias), constituyéndose en representaciones lingüísticas externas presentes en textos y lenguajes como constructos construidos socialmente.

Bases Epistemológicas: Son asumidas desde el pragmatismo en tanto que los cuerpos de conocimiento (como lo son los conceptos) deben ser reconocidos entera 0 parcialmente en términos de su eficacia pragmática, es decir, que para juzgar la eficacia pragmática de diferentes conceptos se debe entender que estas están enfocadas a un problema concreto y a unas circunstancias específicas (contexto).

Es así, como desde esta base epistemológica, la teoría de los PC pretende modelar la heterogeneidad de pensamiento sobre un concepto, comprendiendo las diferentes zonas que exhibe un PC, las cuales despliegan su eficacia pragmática dependiendo de los contextos de uso.

Bases metodológicas: la teoría de los PC se basa en la máxima vygotskyana que establece que para comprender un concepto se debe acudir a la génesis del mismo, lo 
Bio - grafía. Escritos sobre la Biología y su Enseñanza. ISSN 2027-1034

Edición Extraordinaria. p.p. $771-779$

Memorias del IX Encuentro Nacional de Experiencias en Enseñanza de la Biología y la

Educación Ambiental. IV Congreso Nacional de Investigación en Enseñanza de la Biología.

cual es posible con el estudio de los diferentes dominios genéticos que comprenden el concepto (Vygotsky 1978).

Desde esta perspectiva, la teoría de PC adapta los presupuestos de Vygotsky y propone tres dominios genéticos que permiten la comprensión de un concepto; primero, la dimensión socio-cultural del concepto, en la que aludiendo a referentes históricos y epistemológicos se argumenta como se ha desarrollado el concepto, segundo; en la llamada dimensión ontogenética, se propone revisar la literatura existente sobre investigaciones relacionadas a concepciones alternativas o ideas previas sobre un determinado concepto científico y tercero; la dimensión microgenética en la que construyendo instrumentos como cuestionarios, entrevistas, grabaciones de clases, entre otros se puede indagar ideas, concepciones y lenguaje usado por una muestra de personas sobre un concepto científico. Estas tres dimensiones están en relación dialéctica y permiten la construcción de un PC.

El siguiente gráfico sintetiza las relaciones entre las tres dimensiones
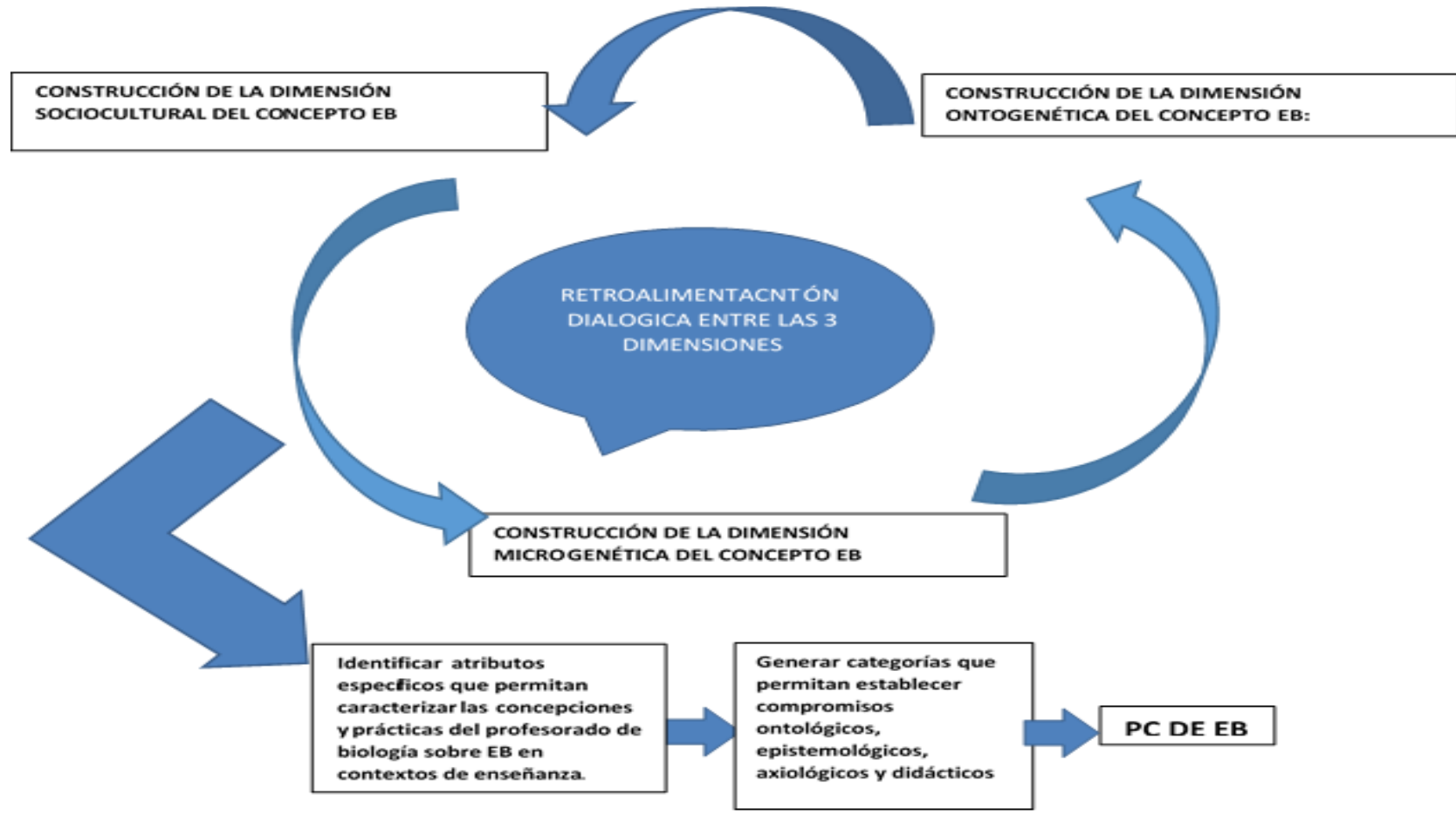

Gráfico 1. Relaciones entre las dimensiones del concepto para construir un PC de EB 
Bio - grafía. Escritos sobre la Biología y su Enseñanza. ISSN 2027-1034

Edición Extraordinaria. p.p. $771-779$

Memorias del IX Encuentro Nacional de Experiencias en Enseñanza de la Biología y la Educación Ambiental. IV Congreso Nacional de Investigación en Enseñanza de la Biología.

\section{METODOLOGÍA}

Esta investigación se desarrolló a partir del estudio de las concepciones del profesorado al enseñar EB, lo que se constituye en un fenómeno educativo estudiado desde el paradigma cualitativo-interpretativo.

En este contexto, según García-Llamas (2010), el paradigma cualitativo "se centra en la descripción y comprensión de los comportamientos individuales de los sujetos, desde un análisis de la realidad que la contempla en su totalidad" (p. 19).

Este tipo de orientación metodológica de investigación se caracteriza por utilizar diversas técnicas para obtener información y provee el fundamento teórico para su interpretación.

Esta investigación se realizó en una muestra de 40 docentes en ejercicio, que enseñan Biología en educación básica la cual se constituye en un subconjunto de la población: los profesores de Biología en educación básica secundaria.

A continuación se muestra parte del instrumento ${ }^{2}$ de indagación que fue desarrollado por los profesores

TABLA 1: CLAVES DE ANÁLISIS DE LAS IDEAS DEL PROFESORADO SOBRE LA EVOLUCIÓN BIOLÓGICA COMO CONCEPTO CIENTÍFICO

\begin{tabular}{|c|c|c|c|}
\hline Conceptos & Categorías & Objetivos & Preguntas \\
\hline \multirow[t]{2}{*}{$\begin{array}{l}\text { Aspectos } \\
\text { generales } \\
\text { Sobre } \\
\text { evolución } \\
\text { biológica } \\
\text { (EB) }\end{array}$} & Ontología & $\begin{array}{l}\text { Identificar compromisos } \\
\text { ontológicos presentes en } \\
\text { las ideas del profesorado } \\
\text { con el concepto }\end{array}$ & $\begin{array}{l}\text { 1. ¿Qué es EB? } \\
\text { 2. ¿En qué medida la EB es } \\
\text { un hecho? } \\
\text { 3. ¿En qué medida la EB es } \\
\text { una teoría? }\end{array}$ \\
\hline & $\begin{array}{l}\text { Epistemología } \\
\text { e historia }\end{array}$ & $\begin{array}{l}\text { ldentificar compromisos } \\
\text { epistemológicos } \\
\text { presentes en las ideas } \\
\text { del profesorado con el } \\
\text { concepto }\end{array}$ & $\begin{array}{l}\text { 4. ¿Qué conceptos claves } \\
\text { estructuran la teoría de la } \\
\text { EB? } \\
\text { 5. ¿Qué hitos históricos } \\
\text { permitieron el desarrollo de } \\
\text { la EB? } \\
\text { 6. ¿Qué evidencias soportan } \\
\text { la teoría de la EB? }\end{array}$ \\
\hline
\end{tabular}

\footnotetext{
${ }^{2}$ Se muestra una pequeña parte del instrumento debido a las limitaciones y requisitos impuestos para el escrito.
} 
Bio - grafía. Escritos sobre la Biología y su Enseñanza. ISSN 2027-1034

Edición Extraordinaria. p.p. $771-779$

Memorias del IX Encuentro Nacional de Experiencias en Enseñanza de la Biología y la Educación Ambiental. IV Congreso Nacional de Investigación en Enseñanza de la Biología.

\section{RESULTADOS Y DISCUSIÓN}

Los resultados de esta investigación se analizaron desde una adaptación a las zonas de perfil propuestas por Sepúlveda (2009) y que se sintetizan en la tabla 2.

TABLA 2. ZONAS DE PC DEL CONCEPTO EB

\begin{tabular}{|c|c|c|}
\hline ZONADE PC & $\begin{array}{l}\text { COMPROMISOS } \\
\text { EPISTEMOLOGICOS Y Y } \\
\text { ONTOLOGICOS EXPRESADOS POR LOS } \\
\text { DOCENTES }\end{array}$ & $\begin{array}{l}\text { PORCENTAJE } \\
\text { DE } \\
\text { DOCENTES } \\
\text { POR ZONA } \\
\text { DE PC }\end{array}$ \\
\hline $\begin{array}{l}\text { AJUSTE } \\
\text { PROVIDENCIAL }\end{array}$ & $\begin{array}{l}\text { Se considera a la EB como un estado existente } \\
\text { y latente en los organismos. } \\
\text { Se explica la EB desde presupuestos } \\
\text { teleológicos externos e internos. } \\
\text { Se considera la agencia de fuerzas } \\
\text { sobrenaturales para explicar los cambios } \\
\text { generados por la EB }\end{array}$ & $10 \%$ \\
\hline $\begin{array}{l}\text { FUNCIONALISMO } \\
\text { INTRAORGÁNICO }\end{array}$ & $\begin{array}{l}\text { El fenómeno del a EB se expresa como un } \\
\text { ajuste de los organismos a sumedio ambiente. } \\
\text { Se da prelación a las causas próximas en } \\
\text { detrimento de las causas remotas que explican } \\
\text { la EB' } \\
\text { Se considera que existe un fin o propósito en la } \\
\text { EB al interior de los organismos (funcionalismo } \\
\text { intra-orgánico) (Condición teleológica de la EB). }\end{array}$ & $25 \%$ \\
\hline $\begin{array}{l}\text { PERSPECTIVA } \\
\text { TRASFORMACIONAL }\end{array}$ & $\begin{array}{l}\text { Se explican los cambios filogenéticos desde la } \\
\text { acumulación de cambios ontogenéticos. } \\
\text { Se considera que existe una tendencia de los } \\
\text { organismos a irse transformando hacia una } \\
\text { mayor complejidad (progreso evolutivo) }\end{array}$ & $45 \%$ \\
\hline PERSPECTIVA & Se considera a la Selección Natural un factor & $20 \%$ \\
\hline
\end{tabular}

${ }^{3}$ Según Mayr (2009) las causas próximas que explican la EB y en particular la adaptación de los organismos y sus estructuras al medio ambiente están dentro de su fisiología, mientras que las causas remotas tienen que ver con el desarrollo filogenético a lo largo de millones de años. 
Bio - grafía. Escritos sobre la Biología y su Enseñanza. ISSN 2027-1034

Edición Extraordinaria. p.p. $771-779$

Memorias del IX Encuentro Nacional de Experiencias en Enseñanza de la Biología y la Educación Ambiental. IV Congreso Nacional de Investigación en Enseñanza de la Biología.

\begin{tabular}{|l|l|l|}
\hline VARIACIONAL & $\begin{array}{l}\text { clave en la EB. } \\
\text { Se consideran presupuestos de la Teoría } \\
\text { Sintética de la EB. }\end{array}$ \\
$\begin{array}{l}\text { Existe pensamiento poblacional para explicar la } \\
\text { EB. } \\
\text { Se considera al fenómeno de la EB como un } \\
\text { proceso no teleológico. }\end{array}$ & \\
\hline
\end{tabular}

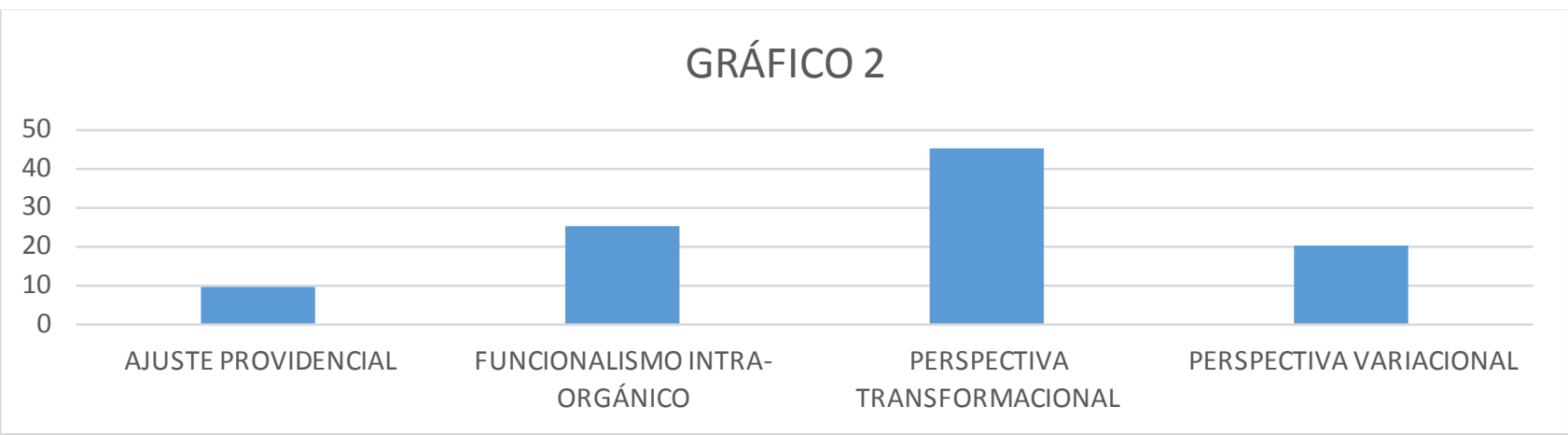

Gráfico 2. Muestra los porcentajes de docentes por cada una de las zonas de perfil establecidas

Los resultados muestran que si bien la zona de PC con menor poder explicativo (ajuste providencial) fue la que presento un menor porcentaje, la mayoría de docentes presentas compromisos epistemológicos y ontológicos que están en zonas de perfil con un poder explicativo de la EB intermedio y que sólo un $20 \%$ de estos expresa compromisos que están en la zona de perfil de mayor poder explicativo de la EB (perspectiva variacional). Las consecuencias didácticas de estos resultados se desarrollarán en el siguiente acápite de este escrito (conclusiones).

\section{CONCLUSIONES}

Los resultados obtenidos en la muestra de docentes analizados revelan que las zonas de perfil presentan una apropiación epistemológica y ontológica del concepto intermedia en cuanto al poder explicativo que se tiene del concepto EB. La toma de conciencia reflexiva

\footnotetext{
${ }^{4}$ La teoría sintética de la EB, es considerada en general la integración de la teoría de la evolución de las especies por la selección natural (Evolución Darwiniana) con la teoría genética como base de la herencia genética, la mutación aleatoria como fuente de variación y la genética de poblaciones.
} 


\section{Bio - grafía. Escritos sobre la Biología y su Enseñanza. ISSN 2027-1034}

Edición Extraordinaria. p.p. 771 - 779

Memorias del IX Encuentro Nacional de Experiencias en Enseñanza de la Biología y la Educación Ambiental. IV Congreso Nacional de Investigación en Enseñanza de la Biología.

de estos compromisos epistemológicos y ontológicos por parte de los docentes puede ser un punto de partida para transformar dichos compromisos hacia zonas de perfil con mayor poder explicativo cómo los de la zona perspectiva variacional. Lo anterior no necesariamente implica que los compromisos epistemológicos y ontológicos de las zonas ajuste providencial y funcionalismo intraorgánico deban ser necesariamente erradicados como se propondría desde los presupuestos de cambio conceptual propuestos por Posner et al. (1982)-, más bien los docentes pueden tomar estos compromisos y desvelar los significados que en torno al concepto de la EB pueden ser útiles para trasegar hacia zonas de perfil más complejas como lo son perspectiva variacional y transformacional, para lo cual es muy útil apropiarse del desarrollo histórico-epistemológico del concepto (dimensión socio-cultural del concepto), ya que si bien, no existe un paralelo exacto entre el desarrollo histórico y epistemológico de un concepto y el aprendizaje o enseñanza del mismo, si se dan algunas relaciones que son importantes tenerlas en cuenta desde el plano didáctico. Por ejemplo, la explicación de la biodiversidad como producto de procesos teleológicos se dio durante un gran periodo de tiempo del pensamiento biológico para después ser transformada hacia el pensamiento evolucionista que no tiene en cuenta las causas ultimas (teleológicas), así mismo en los procesos de enseñanza y aprendizaje del concepto EB podrían aparecer explicaciones teleológicas que luego se podrían transformarse hacia perspectivas evolutivas variacionales no teleológicas (con mayor poder explicativo del fenómeno biodiversidad).

Por otra parte, el reconocimiento de los diferentes compromisos epistemológicos y ontológicos que tienen los docentes con respecto al concepto EB puede convertirse en una valiosa herramienta didáctica para poder comprender las dificultades que tienen los estudiantes para comprender el concepto. En otras palabras, el reconocimiento de las dificultades y obstáculos que tiene el docente (la auto-reflexión) es un punto de partida para mejorar la labor docente.

\section{REFERENCIAS}

Erduran, S., Dagher, Z., Pintó, R., \& Couso, D. (2007). Exemplary Teaching of Argumentation: A Case Study of Two Science Teachers. Contributions from Science Education Research (pág. 403-415): Springer Netherlands

García-Llamas, J. (2010). Métodos y técnicas de investigación en educación. Investigación cualitativa y evaluativa. Madrid: UNED Editorial.

Jiménez, M., \& Díaz de Bustamante, J. (2003). Discurso de aula y argumentación en la clase de ciencias: Cuestiones técnicas y metodológicas. Enseñanza de las Ciencias, 21(3), 359-370.

Mayr, E. (2006). Por qué es única la Biología. Consideraciones sobre la autonomía de una disciplina científica. Katz: Buenos Aires. 280 p.

Mortimer, E. F. (1995). Conceptual change or conceptual profile change? Science \& Education. v. 4, p. 267-285. 
Bio - grafía. Escritos sobre la Biología y su Enseñanza. ISSN 2027-1034

Edición Extraordinaria. p.p. 771 - 779

Memorias del IX Encuentro Nacional de Experiencias en Enseñanza de la Biología y la

Educación Ambiental. IV Congreso Nacional de Investigación en Enseñanza de la Biología.

Mortimer, E. y El-Hani, C. Editors. (2014). Conceptual Profiles A Theory of Teaching and Learning Scientific Concepts. New York: Springer.

Ogborn, J., Kress, G., Martins, I., \& MGillicuddy, K. (1996). Explaining Science in the Classroom. London: Open University Press.

Osborne, J., Erduran, S., \& Simon, S. (2004). Enhancing the quality of argumentation in school science. Journal of Research in Science Teaching, 41(10), 994-1020.

Posner, G. J.; Strike, K. A.; Hewson, P. W. y Gerzog, W. A. (1982). Accommodation of a scientific conception: Toward a theory of conceptual change. En: Science Education (66), 211-227

Sepúlveda, C. y El-Hani, Ch. (2009). Construcción de perfil conceptual de adaptación y análisis de la dinámica discursiva en contextos de enseñanza de la evolución. [Tesis de doctorado]. Salvador: Universidad Federal de Bahía, Facultad de Educación.

Vygotsky, L. S. (1978). Mind in society: The development of higher psychological process. En: M. Cole; V. John-Steiner; S. Scribner y E. Souberman (Eds.). Cambridge, MA: Harvard University Press. 